\title{
ANALOGY BETWEEN MICROPOLAR CONTINUUM AND GRID FRAMEWORKS UNDER INITIAL STRESS
}

\author{
Z. P. Bažant $\dagger$ and M. Christensen $\ddagger$ \\ Department of Civil Engineering, Northwestern University, Evanston, Illinois 60201
}

\begin{abstract}
It is shown that the micropolar medium is a continuum approximation to large grid frameworks under initial axial forces. The strain energy, equilibrium conditions and boundary conditions are formulated, and some inconsistencies in the previous attempts of derivation of the micropolar analogy for gridworks are pointed out. The finite difference method, which is one possible means for the analysis of the overall behavior of a large framework, is investigated and found, by means of numerical examples, to give rather accurate results using much fewer unknowns than the exact analysis. The method is also applicable to problems of overall buckling. It is shown further that the so-called substitute frame in general does not exist, although the finite difference method serves the same purpose. Finally, it is demonstrated that in micropolar continua there exist certain boundary disturbances which rapidly decay with distance from the boundary. A simplified treatment of such disturbances is proposed.
\end{abstract}

\section{INTRODUCTION}

WHEN the methods of frame analysis are applied to the spatial analysis of the tallest buildings presently constructed, a system of over 100,000 algebraic equations with a band width of over 3000 is obtained. With systems of such a large size the capacity of computers presently available is obviously overtaxed. If the fields of dynamics, stability and nonlinear behavior are entered, the size of the system poses difficulties even in the case of plane frameworks.

As a rule, large frames have a regular structure with constant or smoothly variable properties of members. Experience with the exact solutions of the overall behavior of such frames indicates that the displacements of joints vary smoothly from joint to joint. Consequently, the unknown displacements may be grouped together and characterized with a much smaller number of parameters, e.g. by the displacement values in every fifth or tenth joint, assuming that the values at the intermediate joints may be determined with sufficient accuracy by interpolation. This is one method of reducing the size of the system.

In certain cases, especially when the structure is of simple shape, even simpler solutions are possible if the structure is approached as a field problem. This can be done in two ways.

One possibility is to apply the finite difference calculus and obtain exact solutions. This path has been explored for certain cases by many authors, beginning with Bleich and Melan [3]. A detailed survey of this approach can be found in the book by Wah and Calcote [9]; for recent advances along this line consult, e.g. [4].

An alternative possibility is to make a continuous approximation. Generally such an 
idea is quite old; plates and shells have long been used as a model for out-of-plane bending of grillages and lattice shells. However, the theory of a continuum which is suitable as an approximation to the in-plane behavior of a grid framework has been developed only recently. Eringen [5] termed such a continuum micropolar. It represents an extension of the classical Cosserat continuum [7] and a special case of the more general theories of structured continua [5,8]. Its characteristic features are the existence of couple stresses and asymmetric shear stresses, and the independence of microrotation from the displacement field. The possibility of applying these theories to lattice structures and frames was discussed by Wozniak [11] in rather general terms but apparently nothing specific has been presented prior to the paper by Banks and Sokolowski [2]. Their model is not adequate, however, because it represents a Cosserat continuum in which the microrotation and macrorotation are equal, while the corresponding quantities in a frame, that is, the joint rotations and member rotations, are in general unequal. The micropolar continuum as a model for a rectangular gridwork with diagonals was studied by Askar and Cakmak [1]. However, as will be shown later [see equation (9) below], their analogy also appears to be unsatisfactory because all the important terms in the continuous approximation of potential energy have not been included. A similar spatial cubic gridwork with diagonals was investigated by Tauchert [9a] who assumed, similarly as Banks and Sokolowski [2], that for a unit step of grid the couple stresses approximate the couples presented by lattice bars at midspans, in the intersections with the sides of a unit cube centered about the grid point. But this assumption, although seemingly obvious, is not correct, as equation (19) below will demonstrate. The effect of initial stress and buckling of micropolar bodies probably has not yet been treated.

The purpose of this paper is to formulate the analogy between a micropolar medium and a planar uniform rectangular grid framework under initial stress, and to substantiate its applicability by some numerical results.

It must be admitted that an endeavor to formulate a continuum approximation of a discrete system is counter to the current trend. Nevertheless, there exist various cases in which such an approximation is useful. Although the applications will have to be left to a separate paper, a brief mention of the advantages is pertinent. In many high rise buildings, for instance, large grid frameworks interact with continuous bodies, such as stiffening walls, floor slabs or foundations. When the bodies are of rectangular shape, Fourier analysis is evidently the simplest method of solution and can be similarly implemented as in the case of folded plates. For this purpose, the frame must be modelled as a continuum. This approach appears to be much simpler than the alternative of breaking the wall into a discrete system of finite elements and using, for instance, the methods of finite difference calculus for the frame. Even when the frame does not interact with continuous bodies, the continuum approximation of it is advantageous because the finite difference solutions, while being exact and completely analogous to the continuous solutions, lead to more complex expressions. Also, there exist certain cases which admit a simple analytical solution for a continuum while the analogous, discrete, finite difference solution does not satisfy the boundary conditions. Yet another advantage to approximating a frame as a continuum is that the more sophisticated analytical solutions obtained recently for various bodies with couple stresses can then be applied, even if the discrete counterparts of the solutions are not available. Finally, the continuous approximation may be used as a means of transition to a coarser discrete system with a lower number of unknowns. It will be shown that this approach gives an answer to the problem of a substitute frame. 


\section{FRAMEWORK UNDER INITIAL STRESS}

Consider an isolated elastic member of a framework as shown in Fig. 1 which in an unstressed state is parallel to axis $x$ of cartesian axes $x$ and $y$. Let the member be in equilibrium under an initial axial force $P^{0}$ of any magnitude (positive for compression) and

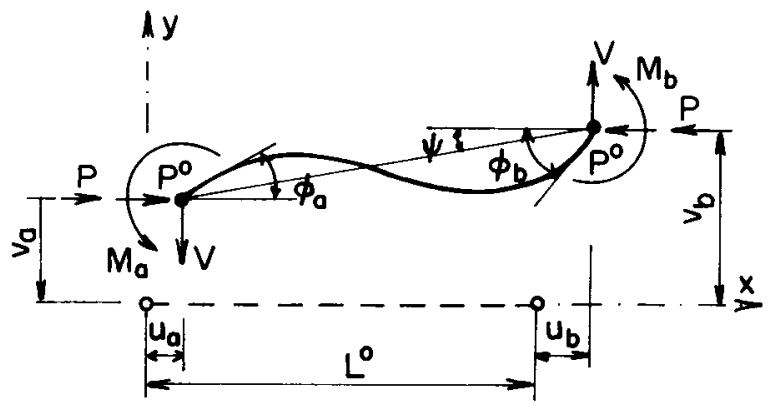

FIG. 1. Positive incremental forces and deformations of a member (assuming the member is parallel to the $x$-axis in its initial stressed state).

initial bending moments and shear forces, $M_{a}^{0}, M_{b}^{0}, V_{a}^{0}, V_{b}^{0}$, acting at the ends $a, b$. Assume that the initial longitudinal and lateral displacements, $u_{a}^{0}, u_{b}^{0}, v_{a}^{0}, v_{b}^{0}$ and the initial end rotations $\varphi_{a}^{0}, \varphi_{b}^{0}$ are small. Further, assume that the member subsequently undergoes an infinitesimal, incremental deformation characterized by small increments in the longitudinal and lateral displacements, $u_{a}, u_{b}, v_{a}, v_{b}$ and small increments in the end rotations, $\varphi_{a}, \varphi_{b}$. The corresponding infinitesimal increments of axial force, bending moments and shear force will be denoted by $P, M_{a}, M_{b}$ and $V$, assuming also that there are no incremental loads between the extremities of the member. The following linearized relationship then holds between the small incremental end reactions and displacements [6]:

$$
\left\{\begin{array}{c}
M_{a} \\
M_{b} \\
P
\end{array}\right\}=\left[\begin{array}{ccc}
k s & k s c & 0 \\
k s c & k s & 0 \\
0 & 0 & E^{\prime}
\end{array}\right]\left\{\begin{array}{c}
\varphi_{a}-\psi \\
\varphi_{b}-\psi \\
u_{a}-u_{b}
\end{array}\right\}
$$

where $\psi=\left(v_{b}-v_{a}\right) / L=$ the counterclockwise rotation of the line $\overline{a b}$ joining the member ends; $L=$ the length of the member in its unstressed state; $k=E I / L ; E^{\prime}=E A / L ; I$ and $A=$ the moment of inertia and area of the cross-section; $E=$ Young's modulus of the material and $s$ and $c$ are the well-known stability functions of the initial axial load $P^{0}$. If the cross-section of the member is constant, $s$ and $c$ are expressed as follows [6]:

$$
\begin{array}{lll}
s=\frac{\alpha(\sin \alpha-\alpha \cos \alpha)}{2-2 \cos \alpha-\alpha \sin \alpha}, & c=\frac{\alpha-\sin \alpha}{\sin \alpha-\alpha \cos \alpha} & \left(P^{0}>0\right) \\
s=\frac{\alpha(\alpha \operatorname{ch} \alpha-\operatorname{sh} \alpha)}{2-2 \operatorname{ch} \alpha+\alpha \operatorname{sh} \alpha}, & c=\frac{\operatorname{sh} \alpha-\alpha}{\alpha \operatorname{ch} \alpha-\operatorname{sh} \alpha} & \left(P^{0}<0\right)
\end{array}
$$


where $\alpha=\pi \sqrt{ }\left(\left|P^{0}\right| / P_{E}\right)=\sqrt{ }\left(\left|P^{0}\right| L / k\right)$ and $P_{E}=\pi^{2} E I / L^{2}$. For $P^{0}=0, s=4, c=\frac{1}{2}$. If the cross-section is variable, the functions $s$ and $c$ may be determined by integrating the differential equation for bending numerically.

The incremental shear force $V$ can be determined from the difference in the moment equilibrium conditions of the member before and after the incremental deformation. When higher order terms are dropped, this leads to the relation

$$
V=-\left(M_{a}+M_{b}\right) / L-P^{0} \psi
$$

Combining this with equation (1) yields:

$$
\left\{\begin{array}{c}
M_{a} \\
M_{b} \\
V \\
P
\end{array}\right\}=\left[\begin{array}{cccc}
k s & k s c & -k s^{\prime} / L & 0 \\
k s c & k s & -k s^{\prime} / L & 0 \\
-k s^{\prime} / L & -k s^{\prime} / L & k s^{\prime \prime} / L^{2} & 0 \\
0 & 0 & 0 & E^{\prime}
\end{array}\right]\left\{\begin{array}{c}
\varphi_{a} \\
\varphi_{b} \\
v_{b}-v_{a} \\
u_{a}-u_{b}
\end{array}\right\}
$$

where

$$
s^{\prime}=s(1+c), \quad s^{\prime \prime}=2 s^{\prime}-\frac{P^{0} L}{k}=2 s^{\prime}-\pi^{2} P^{0} / P_{E}
$$

Consider now a regular rectangular grid framework with members parallel to the cartesian axes $x$ and $y$. In general, the stiffnesses vary from member to member, either because of a change in cross-section or a change in initial axial force. Each joint will be characterized by two numbers, $i$ and $j$ (Fig. 2), increasing in the $x$ - and $y$-directions, respectively. The conditions of equilibrium of joint $(i, j)$ may be expressed using equation (4) to determine the end reactions for each of the four members meeting in the joint. After algebraic rearrangements, the conditions of equilibrium of horizontal forces, vertical



FIG. 2. Numbering of joints and forces acting on a joint. 
forces and moments, can be brought into the following form :

$$
\begin{aligned}
& E_{x}^{\prime}\left(u_{i+1, j}-2 u_{i, j}+u_{i-1, j}\right)+\frac{k_{y} s_{y}^{\prime}}{L_{y}}\left(\varphi_{i, j+1}-\varphi_{i, j-1}\right) \\
& \quad+\frac{k_{y} s_{y}^{\prime \prime}}{L_{y}^{2}}\left(u_{i, j+1}-2 u_{i, j}+u_{i, j-1}\right)+\frac{1}{2} \Delta_{x}\left(E_{x}^{\prime}\right)\left(u_{i+1, j}-u_{i-1, j}\right) \\
& \quad+\frac{1}{2} \Delta_{y}\left(\frac{k_{y} s_{y}^{\prime}}{L_{y}}\right)\left(\varphi_{i, j+1}-2 \varphi_{i, j}+\varphi_{i, j-1}+4 \varphi_{i, j}\right)+\frac{1}{2} \Delta_{y}\left(\frac{k_{y} s_{y}^{\prime \prime}}{L_{y}^{2}}\right)\left(u_{i, j+1}-u_{i, j-1}\right)+f_{x_{i, j}}=0 \\
& E_{y}^{\prime}\left(v_{i, j+1}-2 v_{i, j}+v_{i, j-1}\right)-\frac{k_{x} s_{x}^{\prime}}{L_{x}}\left(\varphi_{i+1, j}-\varphi_{i-1, j}\right) \\
& \quad+\frac{k_{x} s_{x}^{\prime \prime}}{L_{x}^{2}}\left(v_{i+1, j}-2 v_{i, j}+v_{i-1, j}\right)+\frac{1}{2} \Delta_{y}\left(E_{y}^{\prime}\right)\left(v_{i, j+1}-v_{i, j-1}\right) \\
& \quad-\frac{1}{2} \Delta_{x}\left(\frac{k_{x} s_{x}^{\prime}}{L_{x}}\right)\left(\varphi_{i+1, j}-2 \varphi_{i, j}+\varphi_{i-1, j}+4 \varphi_{i, j}\right) \\
& \quad+\frac{1}{2} \Delta_{x}\left(\frac{k_{x} s_{x}^{\prime \prime}}{L_{x}^{2}}\right)\left(v_{i+1, j}-v_{i-1, j}\right)+f_{y_{i, j}}=0 \\
& \quad+\frac{1}{2} \Delta_{y}\left(k_{y} s_{y} c_{y}\right)\left(\varphi_{i, j+1}-\varphi_{i, j-1}\right)+\frac{1}{2} \Delta_{y}\left(\frac{k_{y} s_{y}^{\prime}}{L_{y}}\right)\left(u_{i, j+1}-2 u_{i, j}+u_{i, j-1}\right)-m_{i, j}=0 . \\
& \quad+k_{y} s_{y} c_{y}\left(\varphi_{i, j+1}-2 \varphi_{i, j}+\varphi_{i, j-1}+2 \varphi_{i, j}\right)+2 k_{y} s_{y} \varphi_{i, j}+\frac{k_{y} s_{y}^{\prime}}{L_{y}}\left(u_{i, j+1}-u_{i, j-1}\right) \\
& +\frac{1}{2} \Delta_{x}\left(k_{x} s_{x} c_{x}\right)\left(\varphi_{i+1, j}-\varphi_{i-1, j}\right)-\frac{1}{2} \Delta_{x}\left(\frac{k_{x} s_{x}^{\prime}}{L_{x}}\right)\left(v_{i+1, j}-2 v_{i, j}+v_{i-1, j}\right) \\
& k_{x} s_{x}\left(\varphi_{i+1, j}-2 \varphi_{i, j}+\varphi_{i-1, j}+2 \varphi_{i, j}\right)+2 k_{x} s_{x} \varphi_{i, j}-\frac{k_{x} s_{x}^{\prime}}{L_{x}}\left(v_{i+1, j}-v_{i-1, j}\right) \\
& \\
& \quad
\end{aligned}
$$

Here $u, v=$ joint displacements in the $x$ - and $y$-directions, respectively and $\varphi=$ joint rotation; the subscripts $i$ and $j$, as in $u_{i, j}$ refer to the numbers of the line of vertical members and horizontal members, respectively; subscripts $x, y$ imply quantities which pertain to the members parallel to $x$ or $y ; f_{x}, f_{y}$ and $m$ denote small incremental horizontal load, vertical load and moment applied at the joint and positive in the same sense as the corresponding displacement; and finally, $k_{x}, s_{x}$, etc. and $k_{y}, s_{y}$, etc. represent the average value at joint $(i, j)$ of the corresponding quantity between two adjacent members of the same direction, and $\Delta_{x}(\ldots), \Delta_{y}(\ldots)$ represent the differences of the corresponding quantity between two adjacent members.

Thus, in equations $(5 \mathrm{a})-(5 \mathrm{c})$ the properties of the members $(k, L, E, A)$ as well as their initial axial loads (i.e. $\left.c, s, s^{\prime}, s^{\prime \prime}\right)$ are considered as variable from member to member in both directions.

\section{CONTINUOUS APPROXIMATION}

The finite difference expressions in equations $(5 \mathrm{a})-(5 \mathrm{c})$ are obviously the well-known second order finite difference approximations to the first and second derivatives in a 
rectangular grid whose steps are $L_{x}$ and $L_{y}$, respectively [9]. Equations (5a)-(5c) may thus be approximated by the partial differential equations:

$$
\begin{gathered}
L_{x}^{2}\left(E_{x}^{\prime} u_{, x}\right)_{, x}+2\left(k_{y} s_{y}^{\prime} \varphi\right)_{, y}+\left(k_{y} s_{y}^{\prime \prime} u_{, y}\right)_{, y}+\frac{L_{y}^{2}}{2}\left(k_{y} s_{y}^{\prime}\right)_{, y} \varphi_{, y y}+f_{x}=0 \\
L_{y}^{2}\left(E_{y}^{\prime} v_{, y}\right)_{, y}-2\left(k_{x} s_{x}^{\prime} \varphi\right)_{, x}+\left(k_{x} s_{x}^{\prime \prime} v_{, x}\right)_{, x}-\frac{L_{x}^{2}}{2}\left(k_{x} s_{x}^{\prime}\right)_{, x} \varphi_{, x x}+f_{y}=0 \\
2 k_{x} s_{x}^{\prime}\left(v_{, x}-\varphi\right)-2 k_{y} s_{y}^{\prime}\left(u_{, y}+\varphi\right)-L_{x}^{2}\left(k_{x} s_{x} c_{x} \varphi_{, x}\right)_{, x}-L_{y}^{2}\left(k_{y} s_{y} c_{y} \varphi_{, y}\right)_{, y} \\
+\frac{L_{x}^{2}}{2}\left(k_{x} s_{x}^{\prime}\right)_{, x} v_{, x x}-\frac{L_{y}^{2}}{2}\left(k_{y} s_{y}^{\prime}\right)_{, y} u_{, y y}+m=0 .
\end{gathered}
$$

Here $u, v, \varphi, f_{x}, f_{y}$ and $m$ represent continuous and sufficiently smooth functions of $x$ and $y$ whose values at points $\left(x_{i}, y_{j}\right)$ approximate the values of $u_{i, j}, v_{i, j}$, etc. ; $\dagger$ subscripts $x$ or $y$ following a comma denote partial derivatives, e.g. $v_{, x}=\partial v / \partial x, \varphi_{, x x}=\partial^{2} \varphi / \partial x^{2} ; k_{x}, s_{x}$, $s_{x}^{\prime}, c_{x}, E_{x}^{\prime}$, etc., are also understood as continuous and smooth functions whose values at the midspans of the members approximate the actual member properties. (Introduction of these continuous functions is meaningful only if member properties and initial axial forces vary gradually from member to member. $\ddagger$ )

An alternative approach to obtaining equations (6a)-(6c) can be based on potential energy. The expression for the second order approximation to the incremental strain energy $U_{1}$ for a single member is

$$
U_{1}=\frac{1}{2}\left[M_{a}\left(\varphi_{a}-\psi\right)+M_{b}\left(\varphi_{b}-\psi\right)+P\left(u_{a}-u_{b}\right)\right]-P^{0}\left(L \psi^{2} / 2\right)
$$

plus linear terms $-P^{0}\left(u_{b}-u_{a}\right), M_{a}^{0}\left(\varphi_{a}-\psi\right)$ and $M_{b}^{0}\left(\varphi_{b}-\psi\right)$. As is well known, such terms need not be taken into account since they would yield conditions of equilibrium in the initial stressed state which are assumed to be satisfied. The value $\left(L \psi^{2} / 2\right)$ represents, with an error $0\left(\psi^{4}\right)$, the axial extension of the member due to small lateral displacements $v_{a}, v_{b}$. If $M_{a}, M_{b}$ and $P$ are expressed according to (1), it follows on rearrangement that

$$
U_{1}=\frac{1}{2} E^{\prime}\left(u_{b}-u_{a}\right)^{2}+\frac{1}{2} k s\left(\varphi_{b}-\varphi_{a}\right)^{2}+k s^{\prime}\left(\varphi_{a}-\psi\right)\left(\varphi_{b}-\psi\right)-P^{0} \frac{L \psi^{2}}{2}
$$

The incremental strain energy $U_{x}$ contained in a pair of horizontal members at joint $(i, j)$ is a sum of two expressions of form (8). To avoid lengthy discussion, the properties and initial axial forces of members whose longitudinal axes are in the same line will now be considered as constant. Then, expanding the value of $u, v$ and $\varphi$ in joints $(i+1, j)$ and $(i-1, j)$ in a Taylor's series about the point $(i, j)$ leads to the continuum approximation,

$$
U_{x}=L_{x}^{2} E_{x}^{\prime} u_{, x}^{2}+L_{x}^{2} k_{x} s_{x} \varphi_{, x}^{2}+L_{x}^{2} k_{x} s_{x}^{\prime} \varphi \varphi_{, x x}+2 k_{x} s_{x}^{\prime}\left(\varphi-v_{, x}\right)^{2}-P_{x}^{0} L_{x} v_{, x}^{2} .
$$

In equation (9), only the terms with first derivatives of $u, v$ and $\varphi$ have been retained with the exception of those higher order terms which can be converted on integration by parts

\footnotetext{
$\dagger$ The continuous functions $u, v, \varphi$ may be rigorously defined as the limits of the exact solutions $u_{i, j}, v_{i, j}, \varphi_{i, j}$, for given boundary conditions, as $L_{x}$ and $L_{y}$ in equations $(5 \mathrm{a})-(5 \mathrm{c})$ are considered to tend to zero while the numbers of joints tend to infinity and the coefficients of equations (6a)-(6c) are kept constant.

$\ddagger$ Equations (6), (12), (15) and (19) for constant member properties have already been presented in [13].
} 
to terms containing only first order derivatives. $\dagger$ The term $\varphi \varphi_{, x x}$ is of this type since in the expression for the total energy $\mathscr{U}$ of the structure it can be converted, upon integration by parts, to the term $-\varphi_{, x}^{2}$.

The incremental strain energy $U_{y}$ stored in a pair of vertical members meeting in the joint $(i, j)$ can be similarly obtained. The element of the continuum approximation as shown in Fig. 3 is periodically repeated in both directions. From this it can be seen that the strain energy corresponding to the area $L_{x} L_{y}$ of the frame is $\frac{1}{2}\left(U_{x}+U_{y}\right)$.



FIG. 3. Internal forces at the member midspans and their intuitive analogy with the stresses acting on an element of micropolar continuum.

The total incremental potential energy of the structure, $\mathscr{U}$, is approximated by the expression

$$
\mathscr{U}=\int_{(x)} \int_{(y)}\left[\frac{1}{2}\left(U_{x}+U_{y}\right)-f_{x} u-f_{y} v-m \varphi\right] \frac{\mathrm{d} x \mathrm{~d} y}{L_{x} L_{y}}-W_{B}
$$

where $W_{B}=$ the work of the incremental loads applied at the boundary of the structured body. Integrating the terms involving the products $\varphi \varphi_{, x x}$ and $\varphi \varphi_{, y y}$ by parts (or applying Green's theorem [9]), the integral (10) takes on the form

$$
\mathscr{U}=\int_{(x)} \int_{(y)} U \mathrm{~d} x \mathrm{~d} y-\int_{(x)} \int_{(y)}\left(f_{x} u+f_{y} v+m \varphi\right) \frac{\mathrm{d} x \mathrm{~d} y}{L_{x} L_{y}}-W_{B}^{\prime}
$$

where

$$
\begin{aligned}
U=\{ & L_{x}^{2} E_{x}^{\prime} u_{, x}^{2}+L_{y}^{2} E_{y}^{\prime} v_{, y}^{2}-L_{x}^{2} k_{x} s_{x} c_{x} \varphi_{, x}^{2}-L_{y}^{2} k_{y} s_{y} c_{y} \varphi_{, y}^{2}+2 k_{x} s_{x}^{\prime}\left(\varphi-v_{, x}\right)^{2} \\
& \left.+2 k_{y} s_{y}^{\prime}\left(\varphi+u_{, y}\right)^{2}-P_{x}^{0} L_{x} v_{, x}^{2}-P_{y}^{0} L_{y} u_{, y}^{2}\right\} /\left(2 L_{x} L_{y}\right)
\end{aligned}
$$

and $W_{B}^{\prime}=W_{B}$ plus a certain contour integral of terms involving products $\varphi \varphi_{, x}$ and $\varphi \varphi_{, y}$. Obviously, $U$ can be regarded as the second order terms of the specific incremental strain energy of the continuum approximating the frame. $\$$

$\dagger$ Terms of this type were not considered by Askar and Cakmak [1]. It can be verified that without these terms an agreement with the equilibrium equations based on forces acting on a joint cannot be reached.

$\ddagger$ The presence of negative terms involving $-\varphi_{, x}^{2}$ and $-\varphi_{, y}^{2}$ in equation (12) or terms such as $\varphi \varphi_{, x x}$ and $\varphi \varphi_{, y y}$ in equation (9) that can also become negative raises questions regarding the conditions for positive definiteness of the total potential energy. Such conditions must always be met if $P_{x}^{0}=P_{y}^{0}=0$. That this is indeed so follows from the positive definiteness of expression (8) for $P_{x}^{0}=P_{y}^{0}=0$. 
The differential equilibrium equations may be derived from the first variation of the incremental potential $\mathscr{U}$. This is (without first order terms) given by

$$
\begin{aligned}
\delta \mathscr{U}= & \int_{x} \int_{y}\left\{L_{x}^{2} E_{x}^{\prime} u_{, x} \delta u_{, x}+L_{y}^{2} E_{y}^{\prime} v_{, y} \delta v_{, y}-L_{x}^{2} k_{x} s_{x} c_{x} \varphi_{, x} \delta \varphi_{, x}\right. \\
& -L_{y}^{2} k_{y} s_{y} c_{y} \varphi_{, y} \delta \varphi_{, y}+2 k_{x} s_{x}^{\prime}\left(\varphi-v_{, x}\right)\left(\delta \varphi-\delta v_{, x}\right) \\
& +2 k_{y} s_{y}^{\prime}\left(\varphi+u_{, y}\right)\left(\delta \varphi+\delta u_{, y}\right)-P_{x}^{0} L_{x} v_{, x} \delta v_{, x}-P_{y}^{0} L_{y} u_{, y} \delta u_{, y} \\
& \left.-f_{x} \delta u-f_{y} \delta v-m \delta \varphi\right\} \frac{\mathrm{d} x \mathrm{~d} y}{L_{x} L_{y}}
\end{aligned}
$$

plus a certain contour integral which is relevant only for the boundary conditions. If the terms containing derivatives of the variations are integrated by parts (or the Green's theorem is used) and the equilibrium condition that $\delta \mathscr{U}=0$ for any $\delta u, \delta v, \delta \varphi$ is applied, one obtains the differential equations of equilibrium which are identical with equations $(6 a)-(6 c)$ for constant member properties, as expected.

If the incremental properties of members and initial axial forces are variables from member to member, additional terms must be included in the strain energy density.

* Proceeding in the same manner as from equation (11) to equations (13) and (6a) (6c), it can be verified that in order to obtain the equilibrium equations (6) for the case of variable properties, the strain energy density must be given by the expression (12) plus the term $\dagger$

$$
\left[L_{x}^{2}\left(k_{x} s_{x}^{\prime}\right)_{, x} \varphi_{, x} v_{, x}-L_{y}^{2}\left(k_{y} s_{y}^{\prime}\right)_{, y} \varphi_{, y} u_{, y}\right] /\left(2 L_{x} L_{y}\right)
$$

\section{RELATION OF THE DISCRETE AND CONTINUOUS MODEL AND BOUNDARY CONDITIONS}

A micropolar continuum was defined by Eringen [5] as a continuum whose potential energy density depends, in the plane case, on six independent variables $u_{, x}, v_{, y}, \gamma,(\omega-\varphi)$, $\varphi_{, x}, \varphi_{, y}$ where $\gamma=2 \varepsilon_{x y}=v_{, x}+u_{, y}$ and $\omega=\frac{1}{2}\left(v_{, x}-u_{, y}\right)$. Alternatively, $\gamma$ and $(\omega-\varphi)$ can be replaced by variables $r_{x}=\frac{1}{2} \gamma+(\omega-\varphi)=v_{, x}-\varphi$ and $r_{y}=\frac{1}{2} \gamma-(\omega-\varphi)=u_{, y}+\varphi$. Thus it is seen that $U\left(u_{, x}, v_{, y}, r_{x}, r_{y}, \varphi_{, x}, \varphi_{, y}\right)$ as given by equation (12) for constant member stiffnesses represents a micropolar continuum. This continuum is obviously orthotropic.

The stresses $\sigma_{x x}, \sigma_{y y}, \sigma_{x y}, \sigma_{y x}$ and the couple stresses $m_{x z}, m_{y z}$ in the micropolar continuum of constant properties are defined and expressed from equation (12) as follows:

$$
\begin{aligned}
\sigma_{x x}^{0}+\sigma_{x x} & =\partial \bar{U} / \partial u_{, x}=\sigma_{x x}^{0}+E_{x}^{\prime} u_{, x} L_{x} / L_{y} \\
\sigma_{y y}^{0}+\sigma_{y y} & =\partial \bar{U} / \partial v_{, y}=\sigma_{y y}^{0}+E_{y}^{\prime} v_{, y} L_{y} / L_{x} \\
\sigma_{x y} & =\partial \bar{U} / \partial r_{x}=\partial \bar{U} / \partial v_{, x}=\left(k_{x} s_{x}^{\prime \prime} v_{, x}-2 k_{x} s_{x}^{\prime} \varphi\right) /\left(L_{x} L_{y}\right) \\
\sigma_{y x} & =\partial \bar{U} / \partial r_{y}=\partial \bar{U} / \partial u_{, y}=\left(k_{y} s_{y}^{\prime \prime} u_{, y}+2 k_{y} s_{y}^{\prime} \varphi\right) /\left(L_{x} L_{y}\right) \\
m_{x z} & =\partial \bar{U} / \partial \varphi_{, x}=-k_{x} s_{x} c_{x} \varphi_{, x} L_{x} / L_{y} \\
m_{y z} & =\partial \bar{U} / \partial \varphi_{, y}=-k_{y} s_{y} c_{y} \varphi_{, y} L_{y} / L_{x}
\end{aligned}
$$

$\dagger$ The continuous functions for $k_{x}, s_{x}$, etc. in equations (6a)-(6c) were assumed to approximate the actual values for the member by their values in the midspan points. However, to obtain expression (14) from (8), the actual values would have to be approximated by certain average values of continuous functions for $k_{x}, \ldots$ within the interval between the ends of the member. 
where $\bar{U}=U+\sigma_{x x}^{0} u_{, x}+\sigma_{y y}^{0} v_{, y}=$ the total strain energy density and $\sigma_{x x}^{0}=-P_{x}^{0} / L_{y}$, $\sigma_{y y}^{0}=-P_{y}^{0} / L_{x}$ are the initial stresses. [The symmetric part $\sigma_{(x y)}$ and the antisymmetric part $\sigma_{[x y]}$ of the stress tensor $\sigma_{x y}$ can also be expressed directly as $\sigma_{(x y)}=\frac{1}{2} \partial U / \partial \varepsilon_{x y}, \sigma_{[x y]}=$ $\frac{1}{2} \partial U / \partial(\omega-\varphi)$.]

It is also necessary to define the continuum counterparts of the internal forces. These are characterized by the incremental internal forces in the frame members at midspan (Fig. 3) and include axial force $N$, shear force $T$ and bending moment $M$ which will be taken, for reasons of convenience, about the point on the straight line connecting the ends of the member in the deformed position (and not about the point on the deformed neutral axis). According to (4),

$$
\left.\begin{array}{l}
N=E^{\prime}\left(u_{b}-u_{a}\right)=-P \\
V=k\left[s^{\prime \prime}\left(v_{b}-v_{a}\right) / L-s^{\prime}\left(\varphi_{a}+\varphi_{b}\right)\right] / L \\
M=\left(M_{b}-M_{a}\right) / 2=\frac{1}{2} k s(1-c)\left(\varphi_{b}-\varphi_{a}\right)
\end{array}\right\} .
$$

The end moments $M_{a}$ and $M_{b}$ are related to $M, N, V$ by the following:

$$
M_{a}=-M-\left(V+P^{0} \psi\right) L / 2, \quad M_{b}=M-\left(V+P^{0} \psi\right) L / 2 .
$$

If the member properties are constant in the framework, the continuous approximations of equations (16) in the $x$ - and $y$-directions are straightforward and given by:

$$
\left.\begin{array}{ll}
N_{x}=L_{x} E_{x}^{\prime} u_{, x}, & N_{y}=L_{y} E_{y}^{\prime} v_{, y} \\
T_{x}=\left(k_{x} s_{x}^{\prime \prime} v_{, x}-2 k_{x} s_{x}^{\prime} \varphi\right) / L_{x}, & T_{y}=\left(k_{y} s_{y}^{\prime \prime} u_{, y}+2 k_{y} s_{y}^{\prime} \varphi\right) / L_{y} \\
M_{x}=\frac{1}{2} L_{x} k_{x} s_{x}\left(1-c_{x}\right) \varphi_{, x}, & M_{y}=\frac{1}{2} L_{y} k_{y} s_{y}\left(1-c_{y}\right) \varphi_{, y}
\end{array}\right\}
$$

where $N_{x}, N_{y}, T_{x}, T_{y}, M_{x}, M_{y}$ are continuous functions whose values at midspan approximate the internal forces (16), and where $T_{y}$ was taken as $-V_{y}$ so that its positive direction would correspond to the usual continuum convention.

Expressions (15) for the stresses and couple stresses are simply related to the continuous approximations (18) of the internal forces at the midspans,

$$
\begin{array}{lll}
\sigma_{x x}=N_{x} / L_{y} & \sigma_{y y}=N_{y} / L_{x} \\
\sigma_{x y}=T_{x} / L_{y} & \sigma_{y x}=T_{y} / L_{x} \\
m_{x z}=-\frac{2 c_{x} M_{x}}{L_{y}\left(1-c_{x}\right)} & m_{y z}=-\frac{2 c_{y} M_{y}}{L_{x}\left(1-c_{y}\right)} .
\end{array}
$$

It is interesting to note that, while the normal and shear forces are expressed as resultants of normal and shear stresses over lengths $L_{y}$ and $L_{x}$, the bending moments are not equal to the resultants $L_{y} m_{x z}, L_{x} m_{y z}$ of couple stresses $m_{x z}$ and $m_{y z}$. (For a zero axial force $M_{x}=$ $-L_{y} m_{x z} / 2$ and $M_{y}=-L_{x} m_{y z} / 2$, so that even the sign of $M_{x}$ and $m_{x z}$ is opposite.) The analogy which some authors $[2,9 \mathrm{a}]$ based intuitively on the assumption that the midspan bending moments are expressed as resultants of couple stresses, i.e. $M_{x}=L_{y} m_{x z}$ and $M_{y}=L_{x} m_{y z}$, is thus unrealistic. The reason for the inequality of $M_{x}$ and $L_{y} m_{x z}$ consists, roughly speaking, in the fact that the bending moment varies along the member, while $N_{x}$ and $T_{x}$ are constant along the member. Also, the strain energy of the member due to $\varphi_{, x}$ equals $\frac{1}{2} m_{x z} \varphi_{, x}$ rather than $\frac{1}{2}\left(M_{x} / L_{y}\right) \varphi_{, x}$, whereas the energies due to $N_{x}$ and $T_{x}$ equal $\frac{1}{2}\left(N_{x} / L_{y}\right) u_{, x}$, and $\frac{1}{2}\left(T_{x} / L_{y}\right)\left(v_{, x}-\varphi\right)$. 
Finally, the boundary conditions must be defined. At each boundary joint of the framework, one quantity of each of the pairs $\left(u, f_{x}\right),\left(v, f_{y}\right),(\varphi, m)$, must be given. In the case of a continuum approximation, the expressions (18) have an error of higher than second order only if the functions $u, v, \varphi$ and their derivatives are evaluated at the midspan of the beam. Therefore, when the applied loads $f_{x}, f_{y}$, or $m$ at the boundary joint are prescribed, the simplest formulation of the boundary conditions may be achieved in a manner similar to that used in the solution of continuous boundary value problems by the finite difference method. The gridwork is imagined to be extended beyond the boundary and the hypothetical values in the nodes outside the physical boundary are used in such a way that the internal forces at the midspan of the imagined members crossing the boundary transmit the prescribed forces into the actual boundary joints (Fig. 4). Thus, using equations (16) and (17), the conditions on the left vertical and top horizontal boundaries of the framework are,

$$
\begin{aligned}
N_{x} & =-P_{x}^{B} & N_{y} & =-P_{y}^{B} \\
T_{x} & =V_{x}^{B} & T_{y} & =-V_{y}^{B} \\
M_{x} & =M_{x}^{B}+\left(V_{x}^{B}+P_{x}^{0} \psi_{x}\right) \frac{L_{x}}{2} & M_{y} & =-M_{y}^{B}-\left(V_{y}^{B}+P_{y}^{0} \psi_{y}\right) \frac{L_{y}}{2}
\end{aligned}
$$

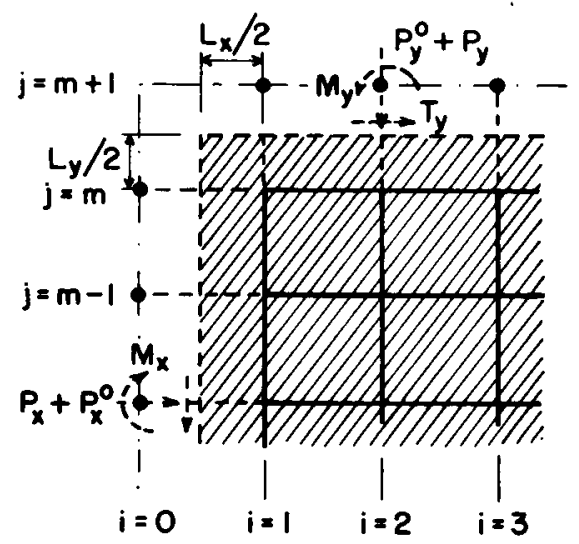

FIG. 4. Fictitious extension of the grid beyond its boundary for the formulation of the free boundary conditions.

where $P^{B}, V^{B}, M^{B}$ denote the prescribed incremental normal load, tangential load and moment, respectively, at the actual boundary joint of the frame. Substituting relations (18), the respective conditions for the left vertical and top horizontal boundaries of the continuum are obtained as follows:

$$
\begin{aligned}
L_{x} E_{x}^{\prime} u_{, x} & =-P_{x}^{B} \\
k_{x} s_{x}^{\prime \prime} v_{, x}-2 k_{x} s_{x}^{\prime} \varphi & =V_{x}^{B} L_{x} \\
L_{x} k_{x} s_{x}\left(1-c_{x}\right) \varphi_{, x} & =2 M_{x}^{B}+\left(V_{x}^{B}+P_{x}^{0} v_{, x}\right) L_{x} \\
L_{y} E_{y}^{\prime} v_{, y} & =-P_{y}^{B} \\
k_{y} s_{y}^{\prime \prime} u_{, y}+2 k_{y} s_{y}^{\prime} \varphi & =-V_{y}^{B} L_{y} \\
L_{y} k_{y} s_{y}\left(1-c_{y}\right) \varphi_{, y} & =-2 M_{y}^{B}-\left(V_{y}^{B}-P_{y}^{0} u_{, y}\right) L_{y} .
\end{aligned}
$$


These conditions are imposed all along the line connecting the midspans of the imagined members crossing the boundary. The continuum boundary can thus be imagined as located beyond the boundary joints of the frame by $L_{x} / 2$ and $L_{y} / 2$, respectively.

\section{FINITE DIFFERENCE METHOD AND THE PROBLEM OF A SUBSTITUTE FRAME}

One possibility for solving the field problem of a continuum is the finite difference method. The form of the finite difference approximations to equations $(6 a)-(6 c)$ as well as the boundary conditions is obvious and will not be written out. The problem is thus converted back to a discrete one. However, by choosing the steps $\Delta x, \Delta y$ of the rectangular grid to be much larger than the distances between the joints, e.g. $\Delta x=5 L_{x}, \Delta y=5 L_{y}$, the number of unknowns is considerably reduced.

Structural analysts [12] have been seeking this objective intuitively, trying to find a substitute frame with greater distances between the joints which would approximate the actual frame. The substitute frame was usually determined from the requirement of equal deflections in a certain typical problem. However, a rational definition of a substitute frame must be based on a requirement that its continuum approximation be the same as for the actual frame. Labeling the quantities for a substitute frame by bars, the condition that the coefficients of all terms of equations $(6 a)-(6 c)$ and the similar finite difference equations with larger steps $\Delta x=\bar{L}_{x}, \Delta y=\bar{L}_{y}$ be equal (or proportional) leads to the relations (in the case of constant properties of all members):

$$
\begin{aligned}
E_{x}^{\prime} L_{x}^{2} & =\beta \bar{E}_{x}^{\prime} \bar{L}_{x}^{2} & E_{y}^{\prime} L_{y}^{2} & =\beta \bar{E}_{y}^{\prime} \bar{L}_{y}^{2} \\
k_{x} s_{x}^{\prime} & =\beta \bar{k}_{x} \bar{s}_{x}^{\prime} & k_{y} s_{y}^{\prime} & =\beta \bar{k}_{y} \bar{s}_{y}^{\prime} \\
k_{x} s_{x}^{\prime \prime} & =\beta \bar{k}_{x} \bar{s}_{x}^{\prime \prime} & k_{y} s_{y}^{\prime \prime} & =\beta \bar{k}_{y} \bar{s}_{y}^{\prime \prime} \\
k_{x} s_{x} c_{x} L_{x}^{2} & =\beta \bar{k}_{x} \bar{s}_{x} \bar{c}_{x} \bar{L}_{x}^{2} & k_{y} s_{y} c_{y} L_{y}^{2} & =\beta \bar{k}_{y} \bar{s}_{y} \bar{c}_{y} \bar{L}_{y}^{2}
\end{aligned}
$$

where $\beta$ is an arbitrary parameter. This is a system of eight equations which, however, involve only five unknowns, namely, $\bar{k}_{x}, \bar{E}_{x}^{\prime}, \bar{k}_{y}, \bar{E}_{y}^{\prime}$ and $\beta$. Therefore a substitute frame in general does not exist. $\dagger$

It should be noted, however, that the finite difference equations approximating the differential equations $(6 a)-(6 c)$ in fact serve the same purpose as a substitute frame. In addition, the finite difference equations have the same form as the equations of a displacement method for a frame would have, and therefore, the existing programs for frames may be easily generalized to include the finite difference solution with steps $\Delta x, \Delta y$ greater than $L_{x}, L_{y}$. When one substitutes $\Delta x=L_{x}, \Delta y=L_{y}$ into such a program, the exact solution is obtained, while for $\Delta x>L_{x}, \Delta y>L_{y}$ an approximate solution is obtained.

In tall building frames one has to account for the fact that axial forces and member cross sections gradually decrease in the upward direction. Thus, the finite difference equations with $\Delta x>L_{x}$ and $\Delta y>L_{v}$ for a frame with smoothly variable properties of members $(k, s, c)$ are needed. These may be obtained directly from equations (5) when the

\footnotetext{
$\dagger$ It should not be inferred from this conclusion that the safety of the existing tall buildings, which all have been analyzed with the help of some substitute frame, is in doubt. Design experience, engineering judgment and intuition has certainly allowed safe designs to be reached even with this approach.
} 
following replacements are made in them:

$$
\left.\begin{array}{rl}
u_{i, j+1}-u_{i, j-1} & \rightarrow\left(u_{i, j+1}-u_{i, j-1}\right) L_{y} / \Delta y, \\
u_{i, j+1}-2 u_{i, j}+u_{i, j-1} & \rightarrow\left(u_{i, j+1}-2 u_{i, j}+u_{i, j-1}\right) L_{y}^{2} / \Delta y^{2}, \\
\Delta_{y}\left(E_{y}^{\prime}\right) \rightarrow \Delta_{y}\left(E_{y}^{\prime}\right) L_{y} / \Delta y, \text { etc. }
\end{array}\right\}
$$

\section{BOUNDARY DISTURBANCES}

To achieve good accuracy by the finite difference method, especially if the frame is not too large, attention must be paid to certain boundary effects typical for structured media. To elucidate these effects, consider a half-plane $y \geq 0$ filled by a framework in which, in order to avoid complex expressions, it is assumed that $L_{x}=L_{y}=L, k_{x}=$ $k_{y}=k, P_{x}^{0}=P_{y}^{0}=0$. Solutions possessing a translational symmetry along axis $x$ will be investigated. Equation (5b) in this case becomes independent and the difference equations $(5 a),(5 c)$ simplify as follows:

$$
\begin{aligned}
L\left(\varphi_{j+1}-\varphi_{j-1}\right)+2\left(u_{j+1}-2 u_{j}+u_{j-1}\right)+f_{x} L^{2} /(6 k) & =0 \\
3\left(u_{j+1}-u_{j-1}\right)+12 L \varphi_{j}+L\left(\varphi_{j+1}-2 \varphi_{j}+\varphi_{j-1}\right)-m L /(2 k) & =0
\end{aligned}
$$

where the subscripts $i$ have been omitted. Equations (23) represent a system of two linear ordinary second order difference equations with constant coefficients for the vectors $u_{j}$ and $\varphi_{j}[10]$. The solution of the homogeneous equation (i.e. $f_{x}=m=0$ ) may be assumed as $\varphi_{j}=C r^{j}, u_{j}=\overline{C r}^{j}$ where $r, C, \bar{C}$ are certain constants. Substitution into (23) for $f_{x}=$ $m=0$ ) leads to a characteristic equation whose roots are found to be $r=0.0717,13.93$ and double root 1.0 . Including also the particular solution for constant loads $f_{x}$ and $m$ in every joint, the following general solution of equations (23) is obtained:

$$
\begin{aligned}
\varphi_{i, j} & =C_{1} 0.0717^{j}+C_{2} 13.93^{j}+C_{3}+f_{x} L j /(12 k)+m /(24 k) \\
u_{i, j} & =1.156 C_{1} L 0.0717^{j}-2 C_{3} L j+C_{4}-0.578 C_{2} L 13.93^{j}-f_{x} L^{2} j^{2} /(12 k)
\end{aligned}
$$

where $C_{1}, C_{2}, C_{3}, C_{4}$ are arbitrary constants.

As boundary conditions consider first that the joints at the base are fixed against displacement, i.e. $u_{j}=0$ at $j=0$, and the joint rotation is prescribed, i.e. $\varphi=\varphi_{B}$ at $j=0$, while at infinity $(j \rightarrow \infty)$ the internal forces are zero [Fig. 5(a)]. The solution (for $f_{x}=$ $m=0$ ) then becomes

$$
\varphi_{i, j}=\varphi_{B} 0.0717^{j}, \quad u_{i, j}=1 \cdot 156 L \varphi_{B}\left(0 \cdot 0717^{j}-1\right) .
$$

If both rotations and displacements are fixed at the base but at infinity shear force $T$ per joint is applied, the solution is:

$$
\varphi_{i, j}=\left(0.0717^{j}-1\right) L T /(12 k), \quad u_{i, j}=0.0965 L^{2} T\left(0.0717^{j}-1\right) / k-L^{2} T j /(6 k) .
$$

Finally, consider that there is a free surface at the base, with half-length vertical members overlapping below the row of joints number one $(j=1)$, each of which is loaded by a moment $M_{B}$ and horizontal force $T_{B}$ [Fig. 5(b)]. At infinity $(j \rightarrow \infty)$ assume a homogeneous 

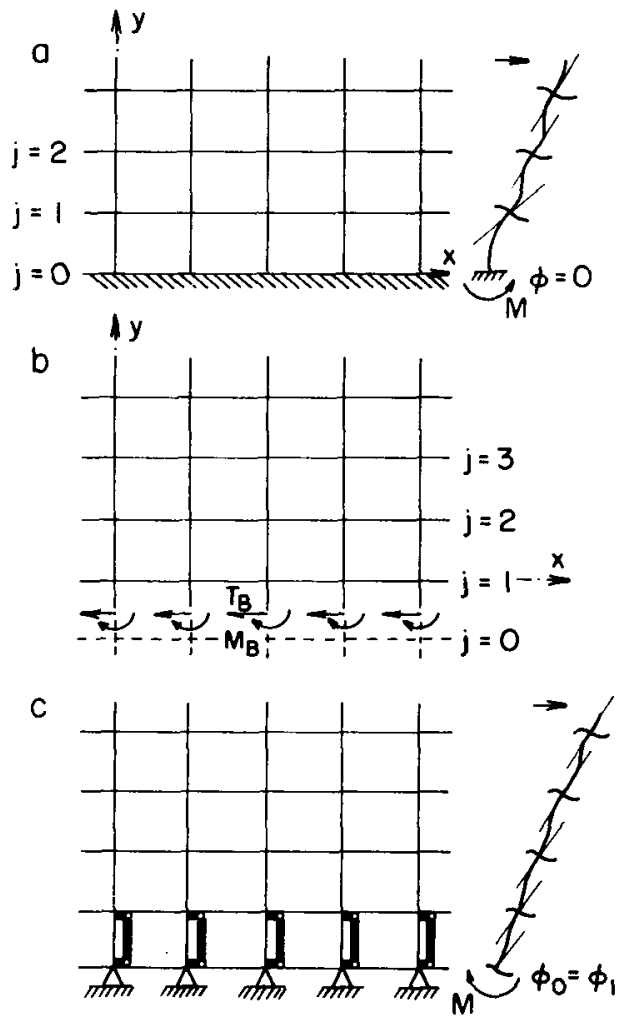

FIG. 5. Boundary conditions (a), (b) giving rise to boundary disturbance and modification (c) of (a) to remove the disturbance.

state of deformation. Note that $T_{y}=-V_{y}$ and $\psi_{y}=-\left(u_{b}-u_{a}\right) / L$. Then, according to equation (16),

$$
T_{B}=12 k\left(u_{1}-u_{0}\right) / L^{2}+6 k\left(\varphi_{0}+\varphi_{1}\right) / L, \quad M_{B}=k\left(\varphi_{1}-\varphi_{0}\right) .
$$

For these boundary conditions, the solution is found to be

$$
\begin{aligned}
\varphi_{i, j} & =-1.078 M_{B} 0 \cdot 0717^{j}-T_{B} L /(12 k) \\
u_{i, j} & =-1.246 M_{B} L 0 \cdot 0717^{j}+T_{B} L^{2} j /(6 k)+C
\end{aligned}
$$

where $C$ is an undetermined constant which requires an additional boundary condition for its evaluation.

An important property of equations (25), (26) and (28) is the rapid decay of the exponential terms with the distance from the boundary. For instance at the second row of joints from the boundary, the exponential terms diminish to 7.2 per cent, at the third row to 0.5 per cent (cf. Fig. 13). For a rectangular gridwork and non-zero axial loads $P_{x}^{0}, P_{y}^{0}$ the solution can be obtained analogously, and rapid decay from the boundary is again found.

If the finite difference method is used, steps longer than $\Delta y=L$ near the boundary would obviously lead to an error of the order of displacement difference between the 
surface and the first row of joints. With the exception of extra large gridworks, such an error should not be committed. Therefore, the first two or three steps $\Delta y$ of the grid near the boundary should be selected equal to $L$. This is not necessary, however, for certain boundary conditions for which the boundary disturbance is absent. From equation (28) it is seen that the boundary disturbance in a half-space under a translationally symmetric deformation disappears if the midspan moment $M_{B}$ is zero, which happens, according to (27), if

$$
\varphi_{1}=\varphi_{0}
$$

is prescribed as the boundary condition (Fig. 5). In the absence of translational symmetry, or for other shapes of the boundary (near corners), the condition for zero disturbance is, of course, somewhat different.

The cumbersome need of using a small step $\Delta y$ equal to $L$ near the boundary may be circumvented by a supe:position technique which is similar to the handling of the edge effects in bending theory of shells. The sharply decaying disturbance is first removed by modifying the boundary conditions. In this case for instance, this may be achieved by imposing the condition of equality for the rotation in the first and the second nodal point from the boundary. The finite difference solution is then obtained using a large grid step throughout, such as $\Delta y=5 L$. Subsequently, on the solution thus obtained, the solution of the boundary disturbance alone, due to the mismatch in $M_{B}$ or $\varphi_{B}$, is superimposed. For an approximate calculation of the disturbance, equations (25) or (28) for a half-space can be used, except in the vicinity of corners. In a general case, the boundary disturbance could be obtained without the use of finite difference calculus by writing the finite difference equilibrium equations $(5 \mathrm{a})-(5 \mathrm{c})$ for only a small region near the surface and solving them as a system of algebraic equations (the interior of the frame being considered as perfectly rigid).

It should be mentioned that the exact solution of the corresponding continuum problem for the boundary disturbance is not a good approximation. The differential equations analogous to difference equations (23) are solved only by trigonometric functions of wavelength $2.57 \mathrm{~L}$ and not any decaying exponentials.

\section{NUMERICAL EXAMPLES}

To explore the accuracy obtainable by the finite difference method with steps $\Delta x, \Delta y$ greater than $L_{x}, L_{y}$, an analysis of a large rectangular plane frame was programmed for computer and the efficiency of various types of solution grid, shown in Figs. 6 and 7, was investigated. One grid was chosen with constant step size; in other grids a reduction of the step size near boundaries was considered. To achieve an accurate representation of the boundary conditions, all of the grids were chosen so as to make the stress boundary of the continuum (the midspan of imaginary members crossing the boundary) coincide with the middle of the grid step. The finite difference approximation of equations $(6 a)-(6 c)$ for varying step size was determined by means of Taylor series expansions. For the sake of simplicity, the properties of members as well as the initial vertical axial loads $P_{y}^{0}$ were considered as constant. The frame was assumed to have 52 floors, 12 column lines, $L_{x}=18 \mathrm{ft}$, $L_{y}=12 \mathrm{ft}$. The horizontal beams were considered as $16 \mathrm{WF} 40$ standard sections, and the columns as 14 WF 320 sections with 2 cover plates $18 \times 1$ in. (In an exact analysis, this plane frame leads to 1872 equilibrium equations for joints with a band width of 75 .) 


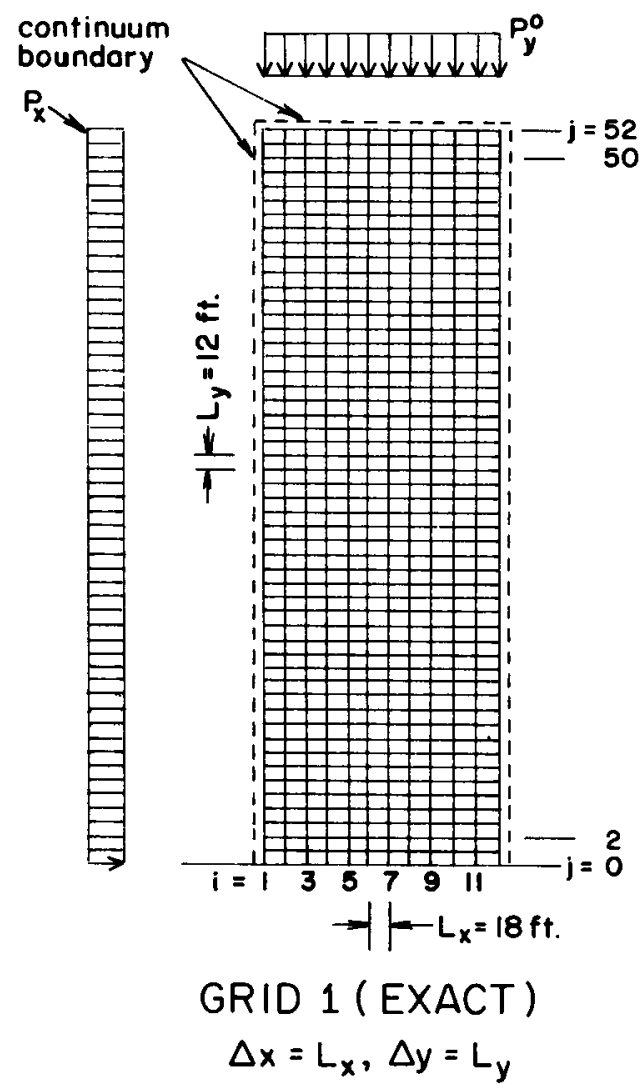

FIG. 6. Frame under initial axial and incremental lateral loads considered in numerical examples and coinciding with the grid for the exact solution.

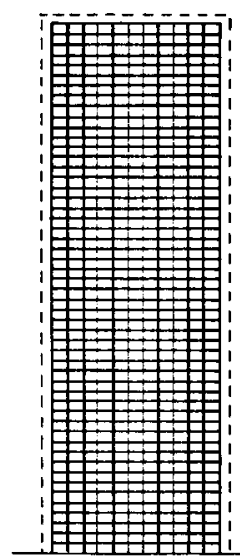

GRID 2

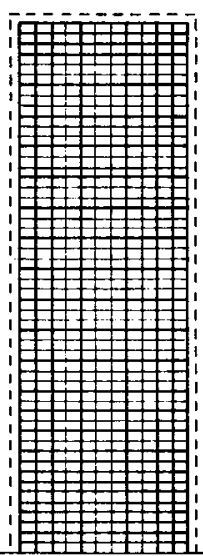

GRID 3

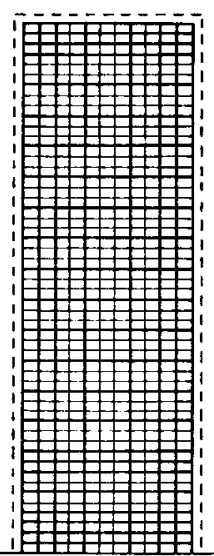

GRID 4

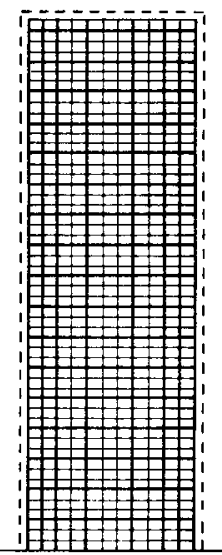

GRID 5

FIG. 7. Grids with a variation in $y$-spacing used for the numerical solution of the finite difference problem for the frame in Fig. 6. 
The solution was carried out for various values of the vertical initial load. The incremental load was considered as a constant horizontal distributed load on the left boundary, $P_{x}=30 L_{x} \mathrm{lb} / \mathrm{ft}$. The results of the analyses obtained with various grids are shown in Figs. 8 and 9. It is seen that grids with a coarse step within the frame give quite accurate results, provided that the first three rows of nodal points near the vertical boundaries are chosen to coincide with the joints of the frame. The results are sufficiently accurate for small as well as large initial axial loads, so that this method may also be used for determination of the buckling of the frame as a whole.

In addition to the frame rigidly supported at the base, analyses have also been carried out for a frame whose boundary condition at the base was modified by releasing the rotation restraint but prescribing equal rotations with the second row of grid joints [equation (29)]. For this boundary condition the boundary disturbance at the base is minimized, and, away from the base, a grid with a large step throughout (grid 5, Fig. 7) does indeed give results very close to the exact solution, as is seen in Fig. 13. The solution for the rigidbase boundary condition may then be approximately obtained by superimposing the solution for the boundary disturbance in a half-space obtained by finite difference calculus, equation (25).

It should be noted that in the typical case when the axial forces and member properties vary in the vertical direction, substitution (22a) yields finite difference equations which are only slightly more complicated than in the above case and can be solved with almost equal ease.

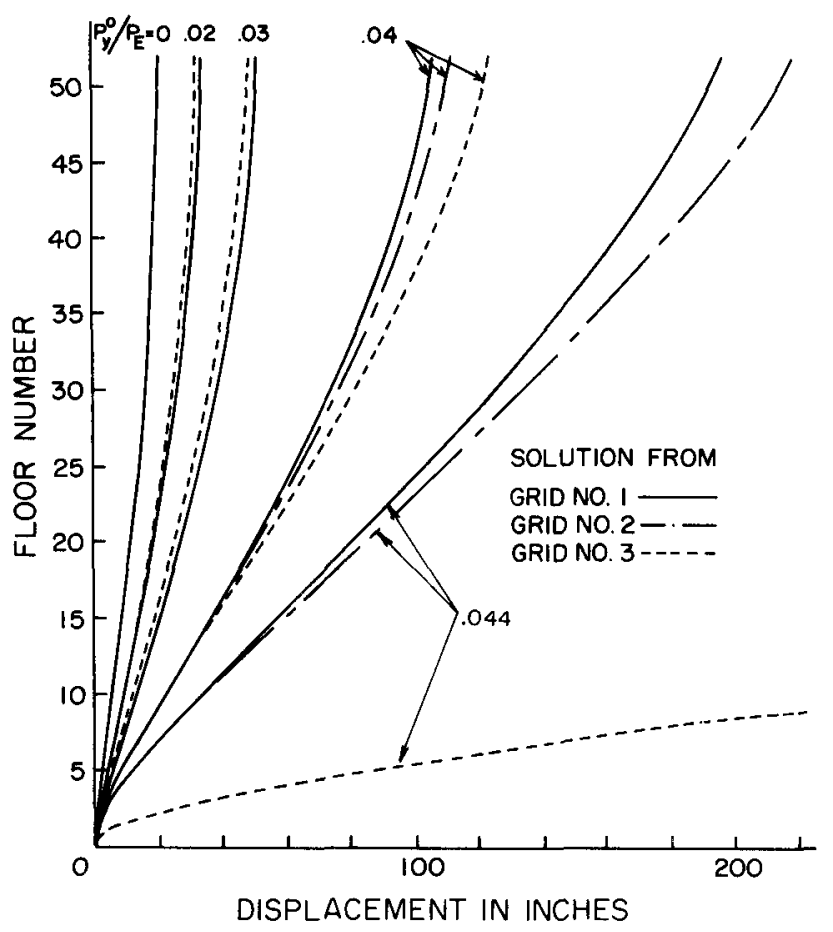

FIG. 8. Horizontal displacement of column line (Fig. 6) under increasing initiai load $P_{y}^{0}$. 


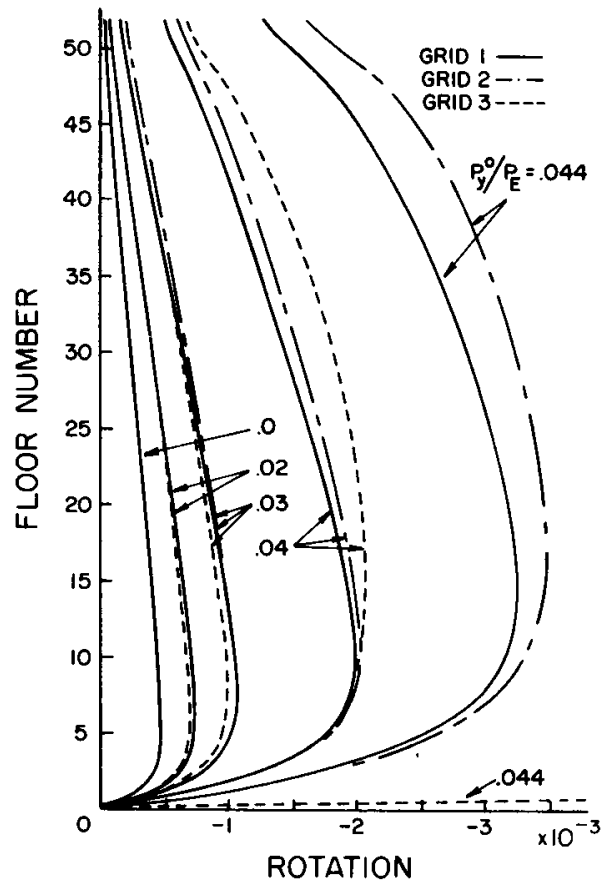

Fig. 9. Joint rotation along column line 1 (Fig. 6) under increasing initial load $P_{y}^{0}$.

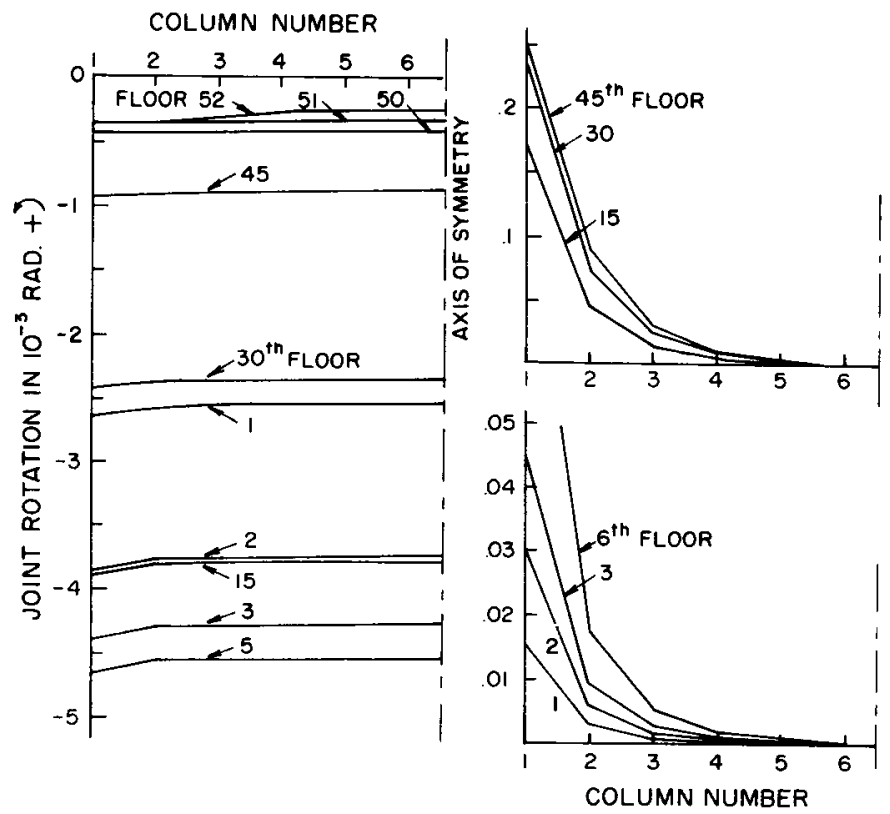

Fig. 10. Exact numerical solution for the distribution of joint rotation and vertical displacement (Fig. 6) for $P_{y}^{0}=0$. 

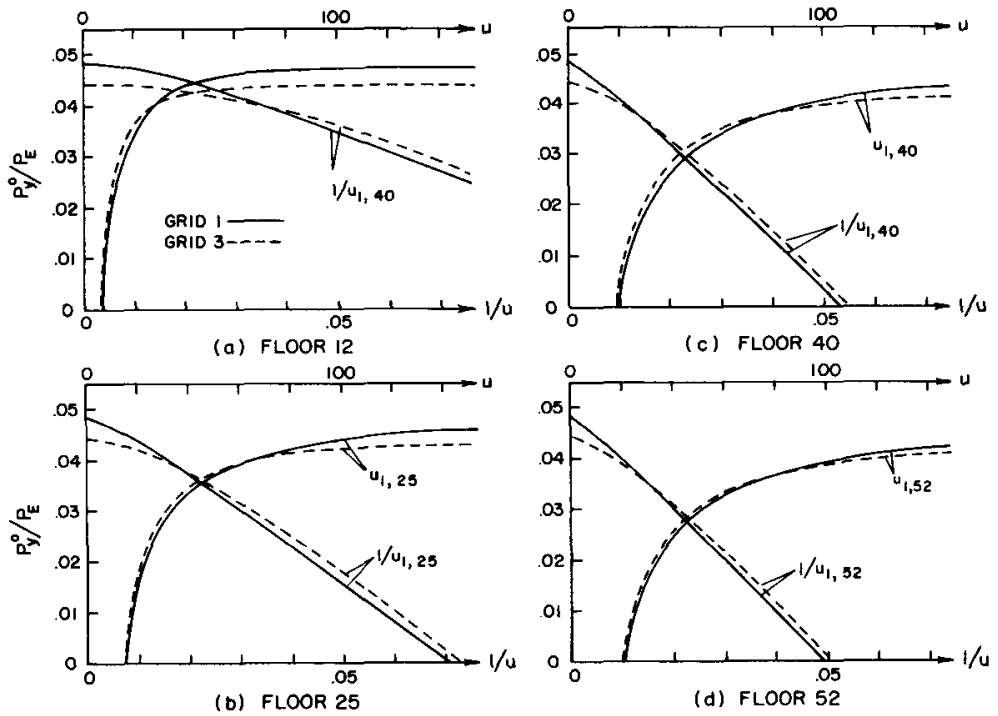

(d) FLOOR 52

FIG. 11. Horizontal displacement of column line 1 (Fig. 6) at various floor loads for increasing initial load $P_{y}^{0}$.
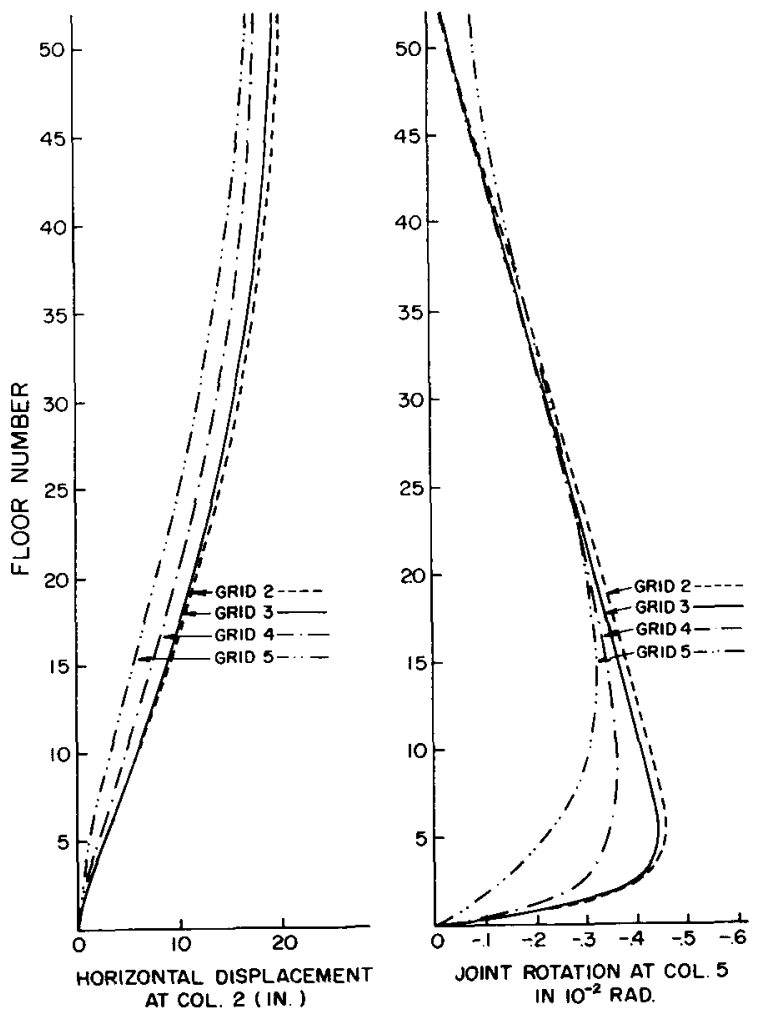

FIG. 12. Comparison of approximate solutions for the frame in Fig. 6 using the grids of Fig. 7. 



Fig. 13. Comparison of approximate solutions for the frame in Fig. 6 with boundary disturbance present and removed and $P_{y}^{0}=0$.

\section{CONCLUSIONS}

1. Large grid frameworks under initial axial forces can be approximated by a micropolar continuum. The incremental equations of equilibrium in terms of displacements are given by equations $(6 \mathrm{a})-(6 \mathrm{c})$, the incremental strain energy density is expressed by equations (12) and (14), the stresses and couple stresses are defined by equations (15) and (19), and the boundary conditions for prescribed forces are given by equation (21). The continuous approximation is formulated even for the case of variable member properties and variable initial axial forces.

2. The finite difference method with grid steps larger than the distances between the joints of a frame may be used as an approximate method of solution, substantially reducing the number of unknowns. This has been demonstrated by numerical examples.

3. A substitute frame in general does not exist, but the finite difference method serves the same purpose.

4. In grid frameworks, as well as in micropolar continua, displacements and rotations, in general, vary sharply near the boundary. To obtain accurate results, such boundary disturbances may not be disregarded. Therefore, the first two or three rows of nodal points near the boundary ought to coincide with the joints of the frame. However, by 
lodifying the boundary conditions [e.g. according to (29)], the boundary disturbance can e made small so that the grid step may be kept large throughout the whole body. The sal effect of a change in boundary conditions must then be approximately evaluated nd superimposed.

5. The couple stresses do not approximate the midspan bending moments in a gridwork ith members of unit length but a more complex relation [equation (19)] applies.

6. The continuum approximation of the potential energy of individual members lust be expressed exactly up to terms with second order derivatives of joint rotation ven if these derivatives are not needed for the expression of potential energy of the microolar continuum.

\section{REFERENCES}

.1] A. Askar and A. S. Cakmak, A structural model of a micropolar continuum. Int. J. Engng Sci. 6, 583-589 (1968).

[2] C. B. BANKS and U. Sokolowski, On certain two-dimensional applications of the couple-stress theory. Int. J. Solids Struct. 4, 15-29(1968).

[3] F. Bleich and E. Melan, Die gewöhnlichen und partiellen Differenzengleichungen der Baustatik. Springer (1927).

[4] D. L. Dean and C. P. Ugarte, Field solutions for two-dimensional frameworks. Int. J. Mech. Sci. 10, 315-339 (1968).

[5] C. E. ERINGEN, Linear theory of micropolar elasticity. J. Math. Mech. 15, 909-923 (1966).

[6] M. Z. Horne and W. Merchant, The Stability of Frames. Pergamon Press (1965).

[7] W. T. KoITER, Couple Stresses in the Theory of Elasticity, Koninklijke Akademie van Wettenschappen, Proc., Ser. B (Physical Sciences), Amsterdam, Vol. 67, pp. 17-44 (1964).

[8] R. D. MindLIN, Microstructure in linear elasticity. Archs ration. Mech. Analysis 17, 51-78 (1966).

[9] . K. ReKTOR YS et al., Survey of Applicable Mathematics. Iliffe (1969).

[9a] T. R. TAUCHERT, A Lattice Theory for Representation of Thermoelastic Composite Materials, Recent Advances in Engineering Science, edited by A. C. ERINGEN, Vol. 5, Part I, pp. 325-345 (1969).

[10] T. WAH and L. R. CALCOTE, Structural Analysis by Finite Difference Calculus. Van Nostrand-Reinhold (1970).

[11] C. WoźniaK, Load-carrying structures of the dense lattice type. The plane problem. Archwm Mech. Stosow. 18, 581-597 (1966); see also Bending and stability problems with lattice structures. Archwm Mech. Stosow. 18, $781-796$ (1966).

[12] American Concrete Institute Committee 442, Response of Buildings to Lateral Forces. ACI J. Proc. 68, 81-106 (1971) (paragraph 4.2.4.2 with references); see also B. J. DesCHAPELLES, Analytical model for lateral load effect on buildings. J. Struct. Div. Am. Soc. civ. Engrs. 96, 1025-1048 (1970).

[13] Z. P. BAŽAnt, Micropolar Medium as a Model for Buckling of Grid Frameworks, Developments in Mechanics, Proceedings of the 12th Midwestern Mechanics Conference, Vol. 6, pp. 587-594 (1971).

(Received 18 March 1971; revised 9 August 1971)

Абстракт - Показано, что микрополярная среда является приближением в смысле сплошной среды для больших сетчатых каркасов, под влиянием начальных осевых усилий. Формулируются энергия деформации, условия равновесия и краевые условия. Даются взгляд на некоторые противоречия в предыдуших попытках выводя микрополярной аналогии для сетчатых конструкций. Исследуется метод конечных разностей, который, как единственный, возможен для анализа полного поведения болвшой рамы. На основе числовых примеров, этот метод дает конечно надлежащие разультаты, при использовании, более менее, неизвестных, по сравнению с точным анализом. Метод можно, также, применить к задачам полного выпучивания. Далее указано, что так называемая зквивалентная рама вообще не существует, несмотря на то, что метод конечных разностей отвечает этой же самой сели. На конец, приводится, что в микрополярных сплошных средах существуют некоторые краевые возмущения, которые быстро уменьшаются при отдалении от края. Предлагается упрощенное рассмотрение таких то возмушений. 